\title{
Nonvanishing spin Hall currents in the presence of magnetic impurities
}

\author{
Pei Wang, You-Quan Li, and Xuean Zhao \\ Zhejiang Institute of Modern Physics and Department of Physics, \\ Zhejiang University, Hangzhou 310027, P. R. China
}

(Dated: June 27, 2018)

\begin{abstract}
The intrinsic spin Hall conductivity in a two dimensional electron gas with Rashba spin-orbit coupling is evaluated by taking account of anisotropic coupling between magnetic impurities and itinerant electrons. In our calculation Kubo's linear response formalism is employed and the vertex correction is considered. In the semiclassical limit $\mu \gg 1 / \tau$, a non-vanishing spin Hall conductivity $\sigma_{s H}$ is found to depend on the momentum relaxation time $\tau$, spin-orbit splitting $\Delta$ and the anisotropic coefficient of interaction between itinerant electrons and magnetic impurities. The clean limit of $\sigma_{s H}$ is in the region of $e / 8 \pi \sim e / 6 \pi$, depending on the anisotropic coefficient.

PACS numbers: 72.25.Dc, 72.25.-b, 72.25.Rb
\end{abstract}

\section{INTRODUCTION}

Recently the spin Hall effect (SHE) [1, 2, $\underline{3}, 4,[4,[5,[6,7,[8]$ has attracted much attention due to its potential application in spintronics. In the spin Hall effect, a longitudinal electric field creates a transverse motion of spins with the spin-up and spin-down carriers moving in opposite directions, which leads to a transverse spin current perpendicular to the external electric field. To understand the spin Hall effect, one needs to study the intrinsic spin Hall effect (ISHE) that has been discussed intensively. Theoretically ISHE may exist in the p-type semiconductor [1] and two-dimensional electron gas (2DEG) 2]. Sinova et al. 2] predicted a universal spin Hall conductivity in clean 2DEG as

$$
\sigma_{s H}=\frac{e}{8 \pi} .
$$

From then on, many researchers have devoted to the issue of whether this result can be modified in the presence of impurity. Some authors found that an arbitrarily small concentration of impurities would suppress the spin Hall conductivity to zero due to the vertex corrections 9,10 , 11, 12, 13. . While others [14, 15, 16] argued that the spin Hall conductivity was robust in the presence of disorder, falling to zero only when the lifetime broadening is larger than the spin orbit splitting of the bands, i.e., $1 / \tau>\Delta$. Grimaldi [17] considered the situation of sufficiently low electron density, and found that the vertex corrections no longer suppressed the spin Hall conductivity when the Fermi energy is comparable to or smaller than the spinorbit energy. Other authors revealed that the vanishing of $\sigma_{s H}$ was a peculiar feature of the linear Rashba model. Taking into account of nonlinear momentum dependence of the spin-orbit interaction $\alpha(p)$ [18] or a nonquadratic band spectrum $\varepsilon(p)$ [19], $\sigma_{s H}$ was robust against impurity scattering.

Very recently, an important step was made by Inoue et al. [20] who found the spin Hall conductivity was not zero in the existence of magnetic impurities in the limit of $\Delta \tau \gg 1$. Physically, the acceleration of the electrons by the external electric field modifies the SO-induced pseudomagnetic field such that the spins are tilted out of the 2DEG plane in directions that are opposite for positive and negative lateral momentum states. This corresponds to a flow of $\sigma_{z}=1 / 2$ and $\sigma_{z}=-1 / 2$ spins in opposite directions without a corresponding net charge transport. In the presence of isotropic impurity scattering, the spin Hall current is proportional to the time derivative of the spin polarization [13] which vanishes in a stationary state. Whereas, this relation does not fulfil for magnetic impurities, leaving over an opportunity for nonvanishing spin Hall conductivity. As we are aware, the case considered by Inoue has not been developed to anisotropic model in the semiclassical limit $\mu \gg 1 / \tau$. And Inoue et al. adopted a simplified approximation in calculating the integral of Green's functions. It is therefore obliged to study such kind models and investigate spin Hall conductivity carefully.

In this paper we calculate the spin Hall conductivity for a two-dimensional magnetically disordered Rashbaelectron gas where the magnetic interaction is anisotropic in the limit of large Fermi circle $\mu \gg 1 / \tau, \Delta$. The XXZtype interaction between the magnetic impurity and the electron spin is adopted. Our calculation is carried out by considering the vertex corrections with the help of Kubo's linear response formalism within the self-consistent Born approximation. The paper is organized as follows: In Sec. III we introduce the model by taking into account of magnetic impurities. In Sec. IIII the calculation procedure of the spin Hall conductivity is presented. In Sec.[IV] we give a comparison between our result and the result in other disordered system and discuss the possible reasons that cause the vanishing spin Hall conductivity.

\section{THE MODEL WITH MAGNETIC IMPURITY}

We consider the 2DEG with Rashba spin-orbit coupling in the presence of impurities, whose Hamiltonian consists of two parts $H=H_{0}+V_{\text {dis }}$ with $H_{0}$ the sum of the kinetic and Rashba terms, and $V_{d i s}$ the potential 
caused by impurities. The main contribution of a shortranged magnetic impurity can be described by an interaction between an itinerant electron and a local magnetic moment 21] whose orientation is defined by polar and azimuthal angles $(\theta, \phi)$ (see Fig. 1). A quite general form of such kind interaction is of XXZ type whose second quantization form is given by the following Hamiltonian

$$
\begin{aligned}
V_{d i s}= & \sum_{i=1}^{N} \int d \mathbf{r}^{2} u \delta\left(\mathbf{r}-\mathbf{R}_{i}\right) \\
& \hat{\psi}^{\dagger}(\mathbf{r})\left(\begin{array}{cc}
\gamma \cos \theta_{i} & \sin \theta_{i} e^{-i \phi_{i}} \\
\sin \theta_{i} e^{i \phi_{i}} & -\gamma \cos \theta_{i}
\end{array}\right) \hat{\psi}(\mathbf{r}),
\end{aligned}
$$

where $\left(\mathbf{R}_{i}, \theta_{i}, \phi_{i}\right)$ denote the position and orientation of the $i$ th impurity, $N$ the total number of impurities; $u$ and $\gamma$ refer to, respectively, the strength and the anisotropy of the coupling between the itinerant electrons and the impurities.

$$
\hat{\psi}(r)=\left(\begin{array}{c}
\hat{\psi}_{\uparrow}(r) \\
\hat{\psi}_{\downarrow}(r)
\end{array}\right) .
$$

The interaction becomes isotropic for the special value $\gamma=1$ which corresponds to the model considered by Inoue [20]. We assume that the distribution of impurities is homogeneous and their orientations are isotropic, accordingly,

$$
\begin{aligned}
& P\left(\mathbf{R}_{1} \theta_{1} \phi_{1}, \mathbf{R}_{2} \theta_{2} \phi_{2}, \cdots, \mathbf{R}_{N} \theta_{N} \phi_{N}\right) \\
& =\left(\frac{1}{4 \pi V}\right)^{N} d \mathbf{R}_{1} d \Omega_{1} d \mathbf{R}_{2} d \Omega_{2} \cdots d \mathbf{R}_{N} d \Omega_{N},
\end{aligned}
$$

where $d \Omega_{i}=\sin \theta_{i} d \theta_{i} d \phi_{i}$ and $V$ denotes the area of the 2DEG. Note that the model given by Eq. (1) for magnetic impurity is not reducible to that for nonmagnetic impurity since the matrix between $\hat{\psi}^{\dagger}(\mathbf{r})$ and $\hat{\psi}(\mathbf{r})$ for the former is a traceless matrix while that for the latter is an unit matrix. For a general discussion, the strength of Rashba coupling is momentum-dependent i.e., $H_{0}=\varepsilon(p)+\alpha(p)\left(\sigma_{x} p_{y}-\sigma_{y} p_{x}\right)$. This Hamiltonian $H_{0}$ can be diagonalized to be

$$
\varepsilon_{ \pm}(p)=\varepsilon(p) \mp p \alpha(p),
$$

by the unitary matrix

$$
U(\mathbf{p})=\frac{1}{\sqrt{2}}\left(\begin{array}{cc}
1 & 1 \\
i e^{i \varphi_{\mathbf{p}}} & -i e^{i \varphi_{\mathbf{p}}}
\end{array}\right)
$$

where $\varphi_{\mathbf{p}}$ denotes the angle between the momentum $\mathbf{p}$ and the $x$ axis. The momentum-dependent Rashba coupling has important effects on the spin-Hall conductivity in the presence of nonmagnetic scatterers [18], while it is not relevant to the final result in the present case with magnetic scatterers.

\section{SPIN HALL CONDUCTIVITY}

As is well known that spin angular momentum is not conserved in the presence of spin-orbit coupling, and the

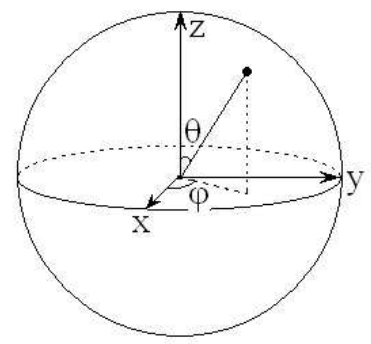

FIG. 1: Bloch sphere representation of an magnetic impurity.

spin current is defined as

$$
\begin{aligned}
J_{y}^{z}(\mathbf{p}) & =\frac{1}{4}\left(v_{y} \sigma_{z}+\sigma_{z} v_{y}\right) \\
& =\frac{1}{2} \frac{d \varepsilon(p)}{d p_{y}} \sigma_{z} .
\end{aligned}
$$

On the basis of Kubo's formalism, the spin Hall conductivity can be expressed as 13]

$$
\begin{aligned}
& \sigma_{s H}(\omega)=\frac{e}{\omega V} \int \frac{d \omega_{1}}{2 \pi} \operatorname{Tr}\left\{n_{F}\left(\omega+\omega_{1}\right) J_{y}^{z}\right. \\
& {\left[G^{r}\left(\omega+\omega_{1}\right)-G^{a}\left(\omega+\omega_{1}\right)\right] j_{x} G^{a}\left(\omega_{1}\right)} \\
& \left.+n_{F}\left(\omega_{1}\right) J_{y}^{z} G^{r}\left(\omega+\omega_{1}\right) j_{x}\left[G^{r}\left(\omega_{1}\right)-G^{a}\left(\omega_{1}\right)\right]\right\}
\end{aligned}
$$

where $n_{F}(\omega)=1 /\left(e^{\beta(\omega-\mu)}+1\right)$ is the Fermi function, $j_{x}(\mathbf{p})=\frac{d}{d p_{x}}\left[\varepsilon(p)+\alpha(p)\left(\sigma_{x} p_{y}-\sigma_{y} p_{x}\right)\right]$ is the one-particle charge current operator, $G^{r}$ and $G^{a}$ are the retarded and advanced Green's functions. The trace is taken over momentum and spin indices. The diagrammatic method is employed to calculate the average spin Hall conductivity over the distribution of impurities. The trace in Eq.(17) is expanded into the sum of diagrams (see Fig. 2).

\section{A. Average Green's functions}

Now we calculate the average Green's functions in the self-consistent Born approximation. For simplicity, the Fermi energy $\mu$ is set to be the zero point of energy since it can be combined in the dispersion relation. Then the bottom of conduction band goes to negative infinite in the large Fermi circle limit. The free Green's function in chiral bases can be expressed as

$$
G_{0(c h)}^{r}(\mathbf{p}, \omega)=\left(\begin{array}{cc}
\frac{1}{\omega-\varepsilon_{+}(p)+i \eta} & 0 \\
0 & \frac{1}{\omega-\varepsilon_{-}(p)+i \eta}
\end{array}\right),
$$

where $\varepsilon_{ \pm}(p)=\varepsilon(p) \mp p \alpha(p)$. The Feynman diagram for the magnetic impurities is shown in Fig. 3. The self-consistent Born equation for the averaged retarded Green's function in chiral bases is given by 


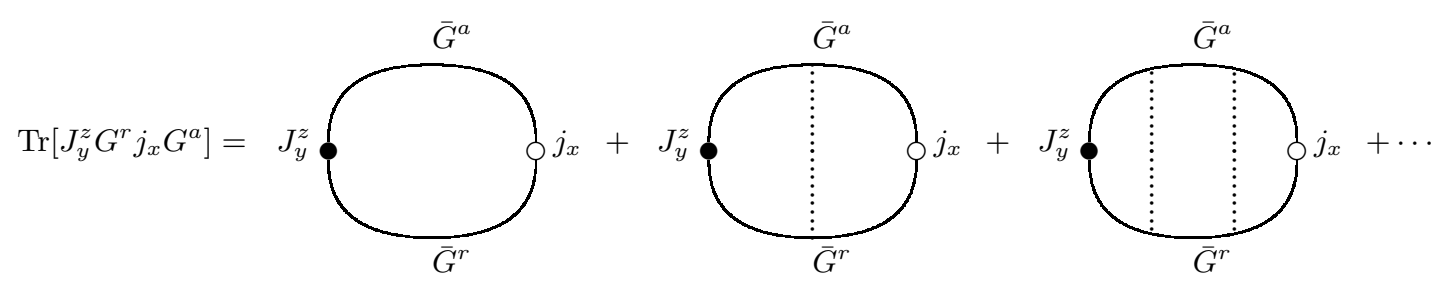

FIG. 2: The spin Hall conductivity is calculated in terms of diagrams. The first diagram contributes to $\sigma_{s H}^{0}$, while the others contribute to the ladder correction for spin Hall conductivity. $\bar{G}^{r}$ and $\bar{G}^{a}$ denote the average Green's functions taking over the distribution of impurities.

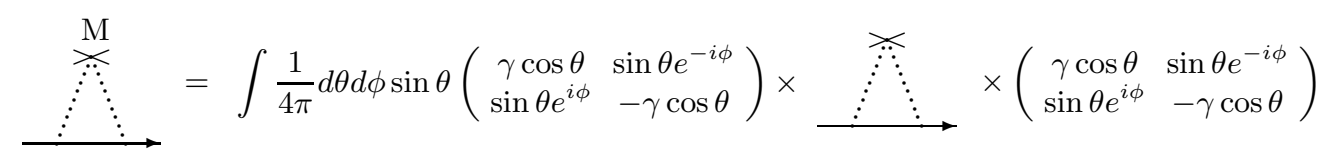

FIG. 3: The Feynman diagram for the magnetic impurity (left hand side of the diagram equation) is different from that for the nonmagnetic impurity (right hand side). Each dot line connected to the same impurity gives an extra matrix and the integration $\int d \theta d \phi \sin \theta / 4 \pi$ for $\theta$ and $\phi$ is performed.

$$
\begin{aligned}
\bar{G}_{(c h)}^{r}(\mathbf{p}, \omega)=G_{0(c h)}^{r}(\mathbf{p}, \omega)+ & \sum_{\mathbf{q}} \int d \theta d \phi \frac{\sin \theta}{4 \pi} \frac{N u^{2}}{V^{2}} G_{0(c h)}^{r}(\mathbf{p}, \omega) U^{\dagger}(\mathbf{p})\left(\begin{array}{cc}
\gamma \cos \theta & \sin \theta e^{-i \phi} \\
\sin \theta e^{i \phi} & -\gamma \cos \theta
\end{array}\right) U(\mathbf{q}) \\
& \bar{G}_{(c h)}^{r}(\mathbf{q}, \omega) U^{\dagger}(\mathbf{q})\left(\begin{array}{cc}
\gamma \cos \theta & \sin \theta e^{-i \phi} \\
\sin \theta e^{i \phi} & -\gamma \cos \theta
\end{array}\right) U(\mathbf{p}) \bar{G}_{(c h)}^{r}(\mathbf{p}, \omega),
\end{aligned}
$$

where $N$ denotes the number of impurities. Eq. (9) has a solution

$\bar{G}_{(c h)}^{r}(\mathbf{p}, \omega)=\left(\begin{array}{cc}\frac{1}{\omega-\varepsilon_{+}(p)+\frac{i}{2 \tau}} & 0 \\ 0 & \frac{1}{\omega-\varepsilon_{-}(p)+\frac{i}{2 \tau}}\end{array}\right)$,

where $\tau$ denotes the momentum-relaxation time and $1 / \tau=n_{i} u^{2} 2 \pi N_{F}\left(\gamma^{2}+2\right) / 3, n_{i}=N / V$ the impurity concentration and $N_{F}$ the density of states at Fermi surface. Similarly, the averaged advanced Green's function is

$$
\bar{G}_{(c h)}^{a}(\mathbf{p}, \omega)=\left(\begin{array}{cc}
\frac{1}{\omega-\varepsilon_{+}(p)-\frac{i}{2 \tau}} & 0 \\
0 & \frac{1}{\omega-\varepsilon_{-}(p)-\frac{i}{2 \tau}}
\end{array}\right) .
$$

The Green's functions in $\sigma_{z}$ bases are

$$
\begin{aligned}
\bar{G}^{r}(\mathbf{p}, \omega) & =U(\mathbf{p}) \bar{G}_{c h}^{r}(\mathbf{p}, \omega) U^{\dagger}(\mathbf{p}), \\
\bar{G}^{a}(\mathbf{p}, \omega) & =U(\mathbf{p}) \bar{G}_{c h}^{a}(\mathbf{p}, \omega) U^{\dagger}(\mathbf{p})
\end{aligned}
$$

Then we calculate $\sigma_{s H}^{0}$, which corresponds to the oneloop diagram in Fig. 2, at zero temperature and zero frequency 


$$
\begin{aligned}
\sigma_{s H}^{0}= & \lim _{\omega \rightarrow 0} \frac{e}{V \omega} \int \frac{d \omega_{1}}{2 \pi} \sum_{\mathbf{p}} \operatorname{Tr}\left\{\theta\left(-\omega-\omega_{1}\right) J_{y}^{z}(\mathbf{p})\left[\bar{G}^{r}\left(\mathbf{p}, \omega+\omega_{1}\right)-\bar{G}^{a}\left(\mathbf{p}, \omega+\omega_{1}\right)\right] j_{x}(\mathbf{p}) \bar{G}^{a}\left(\mathbf{p}, \omega_{1}\right)\right. \\
& \left.+\theta\left(-\omega_{1}\right) J_{y}^{z}(\mathbf{p}) \bar{G}^{r}\left(\mathbf{p}, \omega+\omega_{1}\right) j_{x}(\mathbf{p})\left[\bar{G}^{r}\left(\mathbf{p}, \omega_{1}\right)-\bar{G}^{a}\left(\mathbf{p}, \omega_{1}\right)\right]\right\} \\
= & \frac{e}{8 \pi}\left(1-\frac{1}{1+(\Delta \tau)^{2}}\right),
\end{aligned}
$$

where $\Delta=2 p_{F} \alpha\left(p_{F}\right)$ is the spin-orbit splitting at the Fermi surface, and $\left.p_{F}\right|_{\varepsilon\left(p_{F}\right)=\mu}$ is the Fermi momentum. In deriving the last line of Eq. (13), we took the integral with respect to $\omega_{1}$ and the summation with respect to $\mathbf{p}$ in the limit of large Fermi circle $\mu \gg 1 / \tau, \Delta$ by means of the same method in Ref. 13. Clearly, $\sigma_{s H}^{0}$ is the same as that derived by Dimitrova 13] for nonmagnetic impurities. But it differs from $e / 8 \pi$ derived by Inoue [20], who takes the product of Green functions as a $\delta$-function for simplicity. Such an approximation is valid when $\Delta \tau \gg 1$, but it is inappropriate to take this approximation before taking account of the vertex corrections to the spin Hall conductivity.

\section{B. Vertex correction}

Furthermore, we calculate the vertex correction $\sigma_{s H}^{L}$ to the spin Hall conductivity, which corresponds to the ladder diagram in Fig. 2. Vertex corrections to the terms in Eq.(7) with two advanced or two retarded Green's functions vanish as $\mu \gg 1 / \tau, \Delta[13]$. Only the vertex correction with one advanced and one retarded Green's functions is considered. The sum of ladder diagrams at $\omega=0$ gives

$$
\sigma_{s H}^{L}=\frac{-e}{2 \pi V} \sum_{\mathbf{p}} \operatorname{Tr}\left[\widetilde{J}_{y}^{z} \bar{G}^{r}(\mathbf{p}, 0) j_{x}(\mathbf{p}) \bar{G}^{a}(\mathbf{p}, 0)\right]
$$

where the sum of the series of vertex corrections to the $J_{y}^{z}(\mathbf{p})$ is denoted by the matrix $\widetilde{J}_{y}^{z}$ (see Fig. (4)).

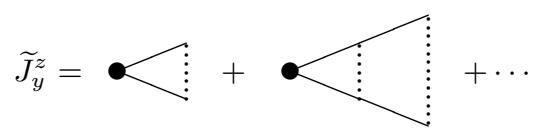

FIG. 4: The vertex of spin current with vertex corrections.

The self-consistent equation of $\widetilde{J}_{y}^{z}$ is

$$
\widetilde{J}_{y}^{z}=\frac{n_{i} u^{2}}{V} \sum_{\mathbf{p}} \int d \theta d \phi \frac{1}{4 \pi} \sin \theta\left(\begin{array}{cc}
\gamma \cos \theta & \sin \theta e^{-i \phi} \\
\sin \theta e^{i \phi} & -\gamma \cos \theta
\end{array}\right) \bar{G}^{a}(\mathbf{p}, 0)\left(J_{y}^{z}(\mathbf{p})+\widetilde{J}_{y}^{z}\right) \bar{G}^{r}(\mathbf{p}, 0)\left(\begin{array}{cc}
\gamma \cos \theta & \sin \theta e^{-i \phi} \\
\sin \theta e^{i \phi} & -\gamma \cos \theta
\end{array}\right) .
$$

In the limit of large Fermi circle $\mu \gg \Delta, 1 / \tau$, the summation over momentum in Eq. (15) can be evaluated by taking integral. As a result, we have

$$
\begin{aligned}
& \left(\widetilde{J}_{y}^{z}\right)_{\uparrow \uparrow}=\left(\frac{\gamma^{2}}{3} B+\frac{2}{3} C\right)\left(\widetilde{J}_{y}^{z}\right)_{\uparrow \uparrow}+\left(\frac{2}{3} B+\frac{\gamma^{2}}{3} C\right)\left(\widetilde{J}_{y}^{z}\right)_{\downarrow \downarrow}, \\
& \left(\widetilde{J}_{y}^{z}\right)_{\downarrow \downarrow}=\left(\frac{2}{3} B+\frac{\gamma^{2}}{3} C\right)\left(\widetilde{J}_{y}^{z}\right)_{\uparrow \uparrow}+\left(\frac{\gamma^{2}}{3} B+\frac{2}{3} C\right)\left(\widetilde{J}_{y}^{z}\right)_{\downarrow \downarrow}, \\
& \left(\widetilde{J}_{y}^{z}\right)_{\uparrow \downarrow}=-\frac{\gamma^{2}}{3} A-\frac{\gamma^{2}}{3} B\left(\widetilde{J}_{y}^{z}\right)_{\uparrow \downarrow}, \\
& \left(\widetilde{J}_{y}^{z}\right)_{\downarrow \uparrow}=\frac{\gamma^{2}}{3} A-\frac{\gamma^{2}}{3} B\left(\widetilde{J}_{y}^{z}\right)_{\downarrow \uparrow},
\end{aligned}
$$

where $v_{F}=d \varepsilon(p) /\left.d p\right|_{\varepsilon(p)=\mu}$ is the Fermi velocity. A, B, and $\mathrm{C}$ are momentum integrals over products of retarded and advanced Green functions.

$$
\begin{aligned}
A & =\frac{-3 i v_{F} \Delta \tau}{4\left(\gamma^{2}+2\right)\left[1+(\Delta \tau)^{2}\right]}, \\
B & =\frac{3}{\gamma^{2}+2}\left(\frac{1}{2}+\frac{1}{2\left[1+(\Delta \tau)^{2}\right]}\right), \\
C & =\frac{3}{\gamma^{2}+2}\left(\frac{1}{2}-\frac{1}{2\left[1+(\Delta \tau)^{2}\right]}\right) .
\end{aligned}
$$

From these equations, we find $\left(\widetilde{J}_{y}^{z}\right)_{\uparrow \uparrow}=\left(\widetilde{J}_{y}^{z}\right)_{\downarrow \downarrow}$ and

$$
\left(\widetilde{J}_{y}^{z}\right)_{\uparrow \downarrow}=-\left(\widetilde{J}_{y}^{z}\right)_{\downarrow \uparrow}=\frac{i v_{F} \Delta \tau}{2+\left(8 / \gamma^{2}+6\right)\left[1+(\Delta \tau)^{2}\right]} .
$$

The vertex correction to the spin Hall conductivity is 
evaluated as

$$
\begin{aligned}
\sigma_{s H}^{L} & =\frac{-i e \Delta \tau\left(\widetilde{J}_{y}^{z}\right)_{\uparrow \downarrow}}{4 \pi v_{F}}\left[1-\frac{1}{1+(\Delta \tau)^{2}}\right] \\
& =\frac{(\Delta \tau)^{2}}{1+\left(4 / \gamma^{2}+3\right)\left[1+(\Delta \tau)^{2}\right]} \sigma_{s H}^{0} .
\end{aligned}
$$

Summing Eq.(13) and Eq.(19), we obtain the spin Hall conductivity

$$
\sigma_{s H}=\sigma_{s H}^{0}+\sigma_{s H}^{L}=\frac{e}{8 \pi} \frac{(\Delta \tau)^{2}}{1+\frac{3 \gamma^{2}+4}{4 \gamma^{2}+4}(\Delta \tau)^{2}} .
$$

This is our main conclusion.

Now we discuss the relation between our work and a recent work on such topics. Inoue et al. 20] considered the vertex corrections due to magnetic impurities, which correspond to the isotropic case $\gamma=1$ of our model. In this special case, our result becomes

$$
\begin{aligned}
\sigma_{s H} & =\left(1+\frac{(\Delta \tau)^{2}}{8+7(\Delta \tau)^{2}}\right) \sigma_{s H}^{0} \\
& =\frac{e}{8 \pi}\left(1+\frac{(\Delta \tau)^{2}}{8+7(\Delta \tau)^{2}}\right)\left(1-\frac{1}{1+(\Delta \tau)^{2}}\right)
\end{aligned}
$$

This differs from the result obtained by Inoue et al. [20]. In their paper, the product of Green functions in Eq. (13) was taken to be a $\delta$-function so that their $\sigma_{s H}^{0}$ and $\sigma_{s H}^{L}$ lack the factor $\left(1-\frac{1}{1+(\Delta \tau)^{2}}\right)$. Such an approximation does not affect the total spin Hall conductivity in the existence of nonmagnetic impurities because the $\sigma_{s H}^{0}$ and $\sigma_{s H}^{L}$ cancel each other. However, the $\sigma_{s H}^{0}$ and $\sigma_{s H}^{L}$ do not cancel each other in the magnetically disordered system, and hence the factor $\left(1-\frac{1}{1+(\Delta \tau)^{2}}\right)$ enters the final result. Even in the situation of $\tau \Delta \gg 1$, the approximation condition of Ref. [20], our $\left.\sigma_{s H}\right|_{\tau \Delta \gg 1}$ does not have the same lower order approximation as that $\frac{e}{8 \pi}\left(1+\frac{(\Delta \tau)^{2}}{8+7(\Delta \tau)^{2}}\right)$ in Ref. [20].

\section{SUMMARY AND DISCUSSION}

Using Kubo's linear-response theory, we have evaluated, in the ladder approximation, the vertex corrections of magnetic impurities on the spin Hall conductivity in a Rashba-split 2DEG. It was assumed that the magnetic impurities were short-ranged and the orientations of their local moments were distributed uniformly. Unlike the case of nonmagnetic impurities, the vertex correction $\sigma_{s H}^{L}$ does not cancel $\sigma_{s H}^{0}$, leading to a non-vanishing spin Hall conductivity (see Eq.(20) ) which depends upon the spinorbit splitting $\Delta$ and the momentum relaxation time $\tau$ in the limit of large Fermi circle $\mu \gg \Delta, 1 / \tau$. The scattering changes both the momentum and spin directions from a magnetic impurity and lead to some correlation between them. Therefore, the average spin tilting cannot cancel the spin current completely. In the dirty limit $\tau \ll 1 / \Delta$, $\sigma_{s H}$ goes to zero. While in the clean limit $\tau \rightarrow \infty$, the spin Hall conductivity is

$$
\lim _{\tau \rightarrow \infty} \sigma_{s H}=\frac{e}{8 \pi} \frac{4 \gamma^{2}+4}{3 \gamma^{2}+4}
$$

which depends on the coefficient $\gamma$. When $\gamma$ changes from 0 to $\infty$, the clean limit of $\sigma_{s H}$ changes from $e / 8 \pi$ to $e / 6 \pi$, and is $e / 7 \pi$ for the isotropic interaction. This result differs from the universal value $e / 8 \pi$ for the ideal clean 2DEG and also differs from the result $\sigma_{s H}=0$ in nonmagnetically disordered system.

Concerning to the vertex corrections, the spin Hall conductivity in nonmagnetically disordered or magnetically disordered systems does not go back to the universal value $e / 8 \pi$ even if the impurity concentration is arbitrarily small. This result comes from the infinite size of system and infinite decoherence length $L_{\varphi}$ assumed in calculation. As $\tau \rightarrow \infty$, the corrected vertex $\left(\widetilde{J}_{y}^{z}\right)_{\uparrow \downarrow}$ goes to zero, but the summation of Green's functions diverges because of lack of a cut-off in momentum space. This divergence leads to a non-vanishing $\sigma_{s H}^{L}$ and changes the total spin Hall conductivity. In real systems, the quantum interference contributing to the vertex corrections only happens inside the decoherence length. So the spin Hall conductivity depends on the decoherence time $\tau_{\varphi}$ when the momentum-relaxation time is large enough to be comparable with it $\tau \sim \tau_{\varphi}$. The $\sigma_{s H}$ derived in this work is only valid when $\tau$ is not too large. Further studies are in progress.

\section{Acknowledgments}

This work was supported by the NSFC No. 10225419, 10674116, 10674117, 60471052; and the Zhejiang Provincial Natural Foundation M603193.
[1] S. Murakami, N. Nagaosa, S. C. Zhang, Science, 301, 1348 (2003).

[2] J. Sinova, D. Culcer, Q. Niu, N. A. Sinitsyn, T. Jung- wirth, A. H. MacDonald, Phys. Rev. Lett., 92, 126603 (2004).

[3] Y. K. Kato, R. C. Myers, A. C. Gossard, D. D. 
Awschalom, Science, 306, 1910 (2004).

[4] J. Wunderlich, B. Kaestner, J. Sinova, T. Jungwirth, Phys. Rev. Lett., 94, 047204 (2005).

[5] H. A. Engel, B. I. Halperin, E. I. Rashba, Phys. Rev. Lett., 95, 166605 (2005).

[6] O. Chalaev, D. Loss, Phys. Rev. B, 71, 245318 (2005).

[7] W. K. Tse, S. DasSarma, Phys. Rev. Lett., 96, 056601 (2006).

[8] S. Zhang, Phys. Rev. Lett., 85, 393 (2000).

[9] E. G. Mishchenko, A. V. Shytov, B. I. Halperin, Phys. Rev. Lett., 93, 226602 (2004).

[10] J. I. Inoue, G. E. W. Bauer, L. W. Molenkamp, Phys. Rev. B, 67, 033104 (2003).

[11] J. I. Inoue, G. E. W. Bauer, L. W. Molenkamp, Phys. Rev. B, 70, 041303(R) (2004).

[12] R. Raimondi, P. Schwab, Phys. Rev. B, 71, 033311 (2005).

[13] O. V. Dimitrova, Phys. Rev. B, 71, 245327 (2005).
[14] J. Schliemann, D. Loss, Phys. Rev. B, 69, 165315 (2004).

[15] A. A. Burkov, A. S. Nunez, A. H. MacDonald, Phys. Rev. B, 70, 155308, (2004).

[16] K. Nomura, J. Sinova, T. Jungwirth, Q. Niu, and A. H. MacDonald, Phys. Rev. B, 71, 041304(R) (2005).

[17] C. Grimaldi, E. Cappelluti, F. Marsiglio, Phys. Rev. B, 73, 081303(R) (2006).

[18] K. Nomura, J. Sinova, N. A. Sinitsyn, A. H. MacDonald, Phys. Rev. B, 72, 165316 (2005).

[19] P. L. Krotkov, S. DasSarma, Phys. Rev. B, 73, 195307 (2006).

[20] J. I. Inoue, T. Kato, Y. Ishikawa, H. Itoh, G. E. W. Bauer, L. W. Molenkamp, Phys. Rev. Lett, 97, 046604 (2006).

[21] P. W. Anderson, J. Phys. C 3, 2436 (1970). K. Yosida, Theory of Magnetism (Springer, New York 1996). 